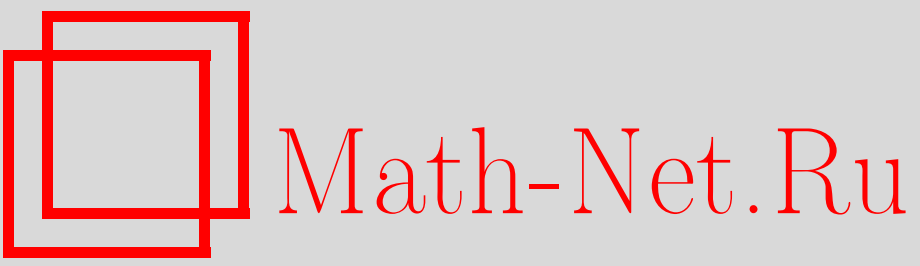

Джао Дзыюнь, $r$-Матрица и алгебро-геометрическое решение системы АКНС, TMФ, 2001, том 127, номер 3, 488-496

DOI: https://doi.org/10.4213/tmf476

Использование Общероссийского математического портала Math-Net.Ru подразумевает, что вы прочитали и согласны с пользовательским соглашением

http://www.mathnet.ru/rus/agreement

Параметры загрузки:

IP : 54.224 .60 .19

26 апреля 2023 г., 13:15:34 
ТЕОРЕТИЧЕСКАЯ

И МАТЕМАТИЧЕСКАЯ

ФИЗИКА

Том 127, № 3

июнь, 2001

(C) 2001 г.

\section{$r$-МАТРИЦА И АЛГЕБРО-ГЕОМЕТРИЧЕСКОЕ РЕШЕНИЕ СИСТЕМЫ АКНС}

Развит подход к конечномерным интегрируемым системам с нелинейными эволюционными уравнениями в рамках $r$-матрицы и алгебро-геометрического решения. Метод иллюстрируется хорошо известным уравнением АКНС. Представлена $r$-матрица АКНС-потока со связями и получено алгебро-геометрическое решение уравнения $\mathrm{AKHC.}$

\section{1. ВВЕДЕНИЕ}

В идеале цель исследования солитонных уравнений, или нелинейных эволюционных уравнений (НЭУ), состоит в получении их явных решений. Уравнения Абловица-Каупа-Ньюэлла-Сегура (АКНС) являются очень важной иерархией НЭУ в теории солитонов [1]. Она может привести к уравнениям Кортевега-де Фриза (КдФ), модифицированному Кд $\Phi$, синус-Гордон, sinh-Гордон, нелинейному уравнению Шредингера и т.п. Все эти уравнения решаются методом обратной задачи рассеяния [2] и обыкновенно имеют $N$-солитонные решения [3]. Одним из направлений исследований в этой области является периодическая задача с граничными условиями, связанная с этими специальными НЭУ. Такую задачу первоначально исследовали Лакс [4] и Дубровин, Кричевер и Новиков [5]. Они использовали блоховские собственные функции и некоторые средства анализа на римановых поверхностях и успешно получили алгебро-геометрические решения (или конечнозонные решения) некоторых хорошо известных нелинейных уравнений, таких как уравнения Кд $\Phi$ и Тоды. Однако алгебро-геометрические решения уравнений AKHC с тех пор не были получены. В настояшей работе мы решаем эту задачу в рамках $r$-матрицы и условия, связывающего конечномерные интегрируемые системы с НЭУ.

Приведем некоторые используемые обозначения: $d p \wedge d q$ обозначает стандартную симплектическую структуру в евклидовом пространстве $R^{2 N}=\left\{(p, q): p=\left(p_{1}, \ldots\right.\right.$ $\left.\left.\ldots, p_{N}\right), q=\left(q_{1}, \ldots, q_{N}\right)\right\}, N>1 ;\langle\cdot, \cdot\rangle$ - стандартное скалярное произведение в $R^{N}$; $[\cdot, \cdot \cdot]$ - обычный коммутатор; $\otimes$ - тензорное произведение двух матриц; $I$ - единичная матрица $2 \times 2$, а $C^{\infty}(R)$ - множество всех $C^{\infty}$-функций на вешественном поле $R$. Скобка

${ }^{*}$ T-7, Theoretical Division, Los Alamos National Laboratory, Los Alamos NM, USA; Institute of Mathematics, Fudan University, Shanghai 200433, P. R. China; Fachbereich 17, Mathematik, Universitaet-Gh Kassel, D-34109 Kassel, Germany. E-mail: zhijun@uni-kassel.de 
Пуассона двух функций Гамильтона $F$ и $G$ на $\left(R^{2 N}, d p \wedge d q\right)$ определена как

$$
\{F, G\}=\sum_{i=1}^{N}\left(\frac{\partial F}{\partial q_{i}} \frac{\partial G}{\partial p_{i}}-\frac{\partial F}{\partial p_{i}} \frac{\partial G}{\partial q_{i}}\right)=\left\langle\frac{\partial F}{\partial q}, \frac{\partial G}{\partial p}\right\rangle-\left\langle\frac{\partial F}{\partial p}, \frac{\partial G}{\partial q}\right\rangle
$$

$\lambda_{1}, \ldots, \lambda_{N}$ суть $N$ произвольным образом заданные различные константы, $\lambda$ и $\mu$ - два различных спектральных параметра, $\Lambda=\operatorname{diag}\left(\lambda_{1}, \ldots, \lambda_{N}\right)$ и

$$
\Gamma_{j}=\sum_{k=1, k \neq j}^{N} \frac{\left(p_{j} q_{k}-p_{k} q_{j}\right)^{2}}{\lambda_{j}-\lambda_{k}}, j=1,2, \ldots, N
$$

\section{2. АКНС-ПОТОК СО СВЯЗЬЮ}

Рассмотрим бесследовую матрицу $2 \times 2$

$$
L=L(\lambda)=\left(\begin{array}{cc}
1 & 0 \\
0 & -1
\end{array}\right)+\sum_{j=1}^{N} \frac{1}{\lambda-\lambda_{j}}\left(\begin{array}{cc}
p_{j} q_{j} & -q_{j}^{2} \\
p_{j}^{2} & -p_{j} q_{j}
\end{array}\right)
$$

которая называется матрицей Лакса. Имеем

$$
\frac{1}{2} \lambda^{2} \operatorname{Tr} L^{2}(\lambda)=\lambda^{2}+2 \lambda\langle p, q\rangle+\langle p, q\rangle^{2}+2 H+\sum_{j=1}^{N} \frac{\lambda_{j}^{2} E_{j}}{\lambda-\lambda_{j}},
$$

где

$$
\begin{aligned}
H & =\langle\Lambda p, q\rangle-\frac{1}{2}\langle q, q\rangle\langle p, p\rangle, \\
E_{j} & =2 p_{j} q_{j}-\Gamma_{j}, \quad j=1, \ldots, N .
\end{aligned}
$$

Конечномерная гамильтонова система, порожденная функцией Гамильтона $H$, имеет Вид

$$
(H):\left\{\begin{array}{l}
q_{x}=\frac{\partial H}{\partial p}=-\langle q, q\rangle p+\Lambda q, \\
p_{x}=-\frac{\partial H}{\partial q}=\langle p, p\rangle q-\Lambda p .
\end{array}\right.
$$

Легко видеть, что система $(H)$ является хорошо известной спектральной задачей Захарова-Шабата (ЗШ) - АКНС [6]

$$
y_{x}=\left(\begin{array}{cc}
\lambda & u \\
v & -\lambda
\end{array}\right) y
$$

со связями

$$
u=-\langle q, q\rangle, \quad v=\langle p, p\rangle,
$$

$\lambda=\lambda_{j}$ и $y=\left(q_{j}, p_{j}\right)^{\mathrm{T}}$. Поэтому мы называем гамильтонову систему $(H)$ вида $(3) A K H C$ потоком с условием (уАКНС); она совпадает с нелинеаризованной АКНС-системой при использовании метода делинеаризации, основанного на парах Лакса [7]. 


\section{3. $r$-МАТРИЦА И ИНТЕГРИРУЕМОСТЬ}

Пусть $L_{1}(\lambda)=L(\lambda) \otimes I$ и $L_{2}(\mu)=I \otimes L(\mu)$. Тогда вьполнена следуюшая теорема.

Теорема 1. Матрица Лакса $L(\lambda)$, определенная уравнением (1), удовлетворяет фундаментальным соотношениям со скобками Пуассона

$$
\{L(\lambda) \stackrel{\otimes}{,} L(\mu)\}=\left[r_{12}(\lambda, \mu), L_{1}(\lambda)\right]-\left[r_{21}(\mu, \lambda), L_{2}(\mu)\right]
$$

где $r_{12}(\lambda, \mu)$ и $r_{21}(\mu, \lambda)$ - стандартнице $r$-матрицы

$$
r_{12}(\lambda, \mu)=\frac{2}{\mu-\lambda} P, \quad r_{21}(\mu, \lambda)=\operatorname{Pr}_{12}(\mu, \lambda) P,
$$

причем

$$
P=\left(\begin{array}{llll}
1 & 0 & 0 & 0 \\
0 & 0 & 1 & 0 \\
0 & 1 & 0 & 0 \\
0 & 0 & 0 & 1
\end{array}\right)
$$

ДокАЗАТЕльСтво. Фундаментальная скобка Пуассона $\left\{L(\lambda),{ }^{\otimes} L(\mu)\right\}$ есть $(4 \times 4)$ матрица [8] с элементами

$$
\{L(\lambda), L(\mu)\}_{k l, m n}=\left\{L(\lambda)_{k m}, L(\mu)_{l n}\right\} .
$$

Прямое вычисление приводит к (5). Матрицы (6) являются $r$-матрицами, поскольку они удовлетворяют уравнению Янга-Бакстера

$$
\left[r_{i j}, r_{i k}\right]+\left[r_{i j}, r_{j k}\right]+\left[r_{k j}, r_{i k}\right]=0, \quad i, j, k=1,2,3 .
$$

Теорема доказана.

ЗАМЕчАНИЕ 1. В действительности, поскольку $r$-матричное соотношение (5) включает только коммутатор, $r$-матрицу $r_{12}(\lambda, \mu)$ можно также выбрать в виде

$$
r_{12}(\lambda, \mu)=\frac{2}{\mu-\lambda} P+I \otimes \widetilde{S}, \quad \widetilde{S}=\left(\begin{array}{ll}
a & b \\
c & d
\end{array}\right),
$$

где элементы $a, b, c$ и $d$ могут быть произвольными функциями $a(\lambda, \mu, p, q), b(\lambda, \mu, p, q)$, $c(\lambda, \mu, p, q)$ и $d(\lambda, \mu, p, q)$, принадлежашими пространству $C^{\infty}(R)$ и зависяшими от спектральных параметров $\lambda$ и $\mu$ и динамических переменных $p$ и $q$. Таким образом, при данной матрице Лакса связанная с ней $r$-матрица определяется не единственным образом. Мы берем простейший случай: $a=b=c=d=0$, т.е. уАКНС-поток имеет стандартную $r$-матрицу (6), которая, очевидно, нединамическая.

Немедленным следствием уравнения (5) является соотношение

$$
\left\{L^{2}(\lambda) \stackrel{\otimes}{,} L^{2}(\mu)\right\}=\left[\bar{r}_{12}(\lambda, \mu), L_{1}(\lambda)\right]-\left[\bar{r}_{21}(\mu, \lambda), L_{2}(\mu)\right],
$$

где

$$
\bar{r}_{i j}(\lambda, \mu)=\sum_{k=0}^{1} \sum_{l=0}^{1} L_{1}^{1-k}(\lambda) L_{2}^{1-l}(\mu) \cdot r_{i j}(\lambda, \mu) \cdot L_{1}^{k}(\lambda) L_{2}^{l}(\mu), \quad i j=12,21 .
$$


Таким образом, уравнение (7) приводит к равенствам

$$
4\left\{\operatorname{Tr} L^{2}(\lambda), \operatorname{Tr} L^{2}(\mu)\right\}=\operatorname{Tr}\left\{L^{2}(\lambda) \stackrel{\otimes}{,} L^{2}(\mu)\right\}=\operatorname{Tr}\left\{L_{1}^{2}(\lambda) \stackrel{\otimes}{,} L_{2}^{2}(\mu)\right\}=0,
$$

гарантирующим инволютивность полученных в (2) интегралов движения. Как следствие имеем

$$
\left\{E_{i}, E_{j}\right\}=\left\{H, E_{j}\right\}=\left\{F_{s}, E_{j}\right\}=0, \quad i, j=1,2, \ldots, N, \quad s=0,1,2, \ldots,
$$

где

$$
F_{s}=\sum_{j=1}^{N} \lambda_{j}^{s} E_{j}=2\left\langle\Lambda^{s} p, q\right\rangle-\sum_{j+k=s-1}\left(\left\langle\Lambda^{j} p, p\right\rangle\left\langle\Lambda^{k} q, q\right\rangle-\left\langle\Lambda^{j} p, q\right\rangle\left\langle\Lambda^{k} q, p\right\rangle\right) .
$$

Кроме того, $E_{1}, E_{2}, \ldots, E_{N}$ функционально независимы на некоторой области в $R^{2 N}$; отсюда получаем следующую теорему.

ТЕОРема 2. Определенный в (3) уАКНС-поток полностью интегрируем в смысле Лиувилля.

ЗАМЕчАНИЕ 2. Интегрируемость уАКНС-потока доказана здесь в рамках $r$-матрицы и матрицы Лакса, а не пары Лакса.

\section{4. ИЕРАРХИЯ АКНС И ЕЕ ИНВОЛЮТИВНОЕ РЕШЕНИЕ}

В данном разделе рассматривается АКНС-иерархия НЭУ. Мы связываем спектральную задачу ЗШ [6] и АКНС-иерархию [2] с конечномерными гамильтоновыми системами $(H)$ и $\left(F_{s}\right), s=0,1, \ldots$. Начнем со спектральной задачи ЗШ $(4)$, где $\lambda$ - собственное значение, $y=\left(y_{1}, y_{2}\right)^{\mathrm{T}}$ - соответствуюшая векторная собственная функция, а $u$ и $v$ - два потенциала, которые или затухают на бесконечности, или имеют периодические граничные условия. Тогда АКНС-иерархия связанных с задачей (4) НЭУ выводится следующим образом:

$$
\left(\begin{array}{l}
u \\
v
\end{array}\right)_{t_{s}}=J G_{s}, \quad s=0,1,2, \ldots,
$$

где $\left\{G_{s}=J^{-1} K G_{s-1}\right\}_{s=0}^{\infty}-$ последовательность Ленарда при $G_{-1}=(0,0)^{\mathrm{T}}$ и $G_{0}=$ $(v, u)^{\mathrm{T}}$, а два симметричных оператора $K$ и $J$ имеют вид

$$
K=\left(\begin{array}{cc}
2 u \partial^{-1} u & \partial-2 u \partial^{-1} v \\
\partial-2 v \partial^{-1} u & -2 v \partial^{-1} v
\end{array}\right), \quad J=2\left(\begin{array}{cc}
0 & -1 \\
1 & 0
\end{array}\right)
$$

$\left(\partial=\partial / \partial x\right.$ и $\left.\partial \partial^{-1}=\partial^{-1} \partial=1\right)$. Показательным представителем иерархии (9) является уравнение при $s=2$ :

$$
u_{t}=-\frac{1}{2} u_{x x}+u^{2} v, \quad v_{t}=\frac{1}{2} v_{x x}-v^{2} u, \quad t=t_{2} .
$$

Рассмотрим функции Гамильтона $F_{s}$, определенные в $(8)$. Из скобки Пуассона $\left\{F_{s}\right.$, $H\}=0, s=0,1, \ldots$, следует, что все канонические гамильтоновы системы $\left(F_{s}\right)$ и $(H)$ полностью интегрируемы по Лиувиллю. Поэтому их гамильтоновы потоки коммутируют между собой.

Пусть $\left(p\left(x, t_{s}\right), q\left(x, t_{s}\right)\right)^{\mathrm{T}}$ - решение согласованных канонических гамильтоновых уравнений $(H)$ и $\left(F_{s}\right)$, называемое инволютивным.м решением [9]. Тогда имеем следующую теорему. 
TEOPEMA 3. Функиии

$$
u=-\left\langle q\left(x, t_{s}\right), q\left(x, t_{s}\right)\right\rangle, \quad v=\left\langle p\left(x, t_{s}\right), p\left(x, t_{s}\right)\right\rangle, \quad s=0,1, \ldots,
$$

удовлетворяют АКНС-уравнениям высших порядков (9). В частности, уравнению (11) удовлетворяет решение

$$
u=-\left\langle q\left(x, t_{2}\right), q\left(x, t_{2}\right)\right\rangle, \quad v=\left\langle p\left(x, t_{2}\right), p\left(x, t_{2}\right)\right\rangle,
$$

где $\left(p\left(x, t_{2}\right), q\left(x, t_{2}\right)\right)^{\mathrm{T}}$ - инволютивное решение согласованных гамильтоновых систем $(H) u\left(F_{2}\right)$.

ДоказАТЕЛЬСТво. Принимая во внимание $(3)$, равенства $q_{t_{s}}=\partial F_{s} / \partial p$ и $p_{t_{s}}=$ $-\partial F_{s} / \partial q$, а также основное равенство

$$
K\left(\begin{array}{c}
\left\langle p\left(x, t_{s}\right), p\left(x, t_{s}\right)\right\rangle \\
-\left\langle q\left(x, t_{s}\right), q\left(x, t_{s}\right)\right\rangle
\end{array}\right)=J\left(\begin{array}{c}
\left\langle\Lambda p\left(x, t_{s}\right), p\left(x, t_{s}\right)\right\rangle \\
-\left\langle\Lambda q\left(x, t_{s}\right), q\left(x, t_{s}\right)\right\rangle
\end{array}\right)
$$

где операторы $K$ и $J$ определены в (10), можно проверить, что АКНС-уравнения высших порядков (9) выполнены для

$$
u=-\left\langle q\left(x, t_{s}\right), q\left(x, t_{s}\right)\right\rangle, \quad v=\left\langle p\left(x, t_{s}\right), p\left(x, t_{s}\right)\right\rangle, \quad s=0,1, \ldots
$$

\section{5. АЛГЕБРО-ГЕОМЕТРИЧЕСКОЕ РЕШЕНИЕ}

В следуюшей процедуре мы получим явное выражение для решения (12), т.е. выведем алгебро-геометрическое решение АКНС-уравнения (11). Для этого перепишем матрицу Лакса (1) в виде

$$
L=\left(\begin{array}{cc}
A(\lambda) & B(\lambda) \\
C(\lambda) & -A(\lambda)
\end{array}\right)
$$

где

$$
A(\lambda)=1+\sum_{j=1}^{N} \frac{1}{\lambda-\lambda_{j}} p_{j} q_{j}, \quad B(\lambda)=-\sum_{j=1}^{N} \frac{1}{\lambda-\lambda_{j}} q_{j}^{2}, \quad C(\lambda)=\sum_{j=1}^{N} \frac{1}{\lambda-\lambda_{j}} p_{j}^{2} .
$$

Можно подставить $B(\lambda)$ и $C(\lambda)$ в виде отношений

$$
B(\lambda) \equiv-\frac{\langle q, q\rangle Q_{B}(\lambda)}{K(\lambda)}, \quad C(\lambda) \equiv \frac{\langle p, p\rangle Q_{C}(\lambda)}{K(\lambda)}
$$

где

$$
\begin{aligned}
& \langle q, q\rangle Q_{B}(\lambda)=\sum_{j=1}^{N} q_{j}^{2} \prod_{k=1, k \neq j}^{N}\left(\lambda-\lambda_{k}\right), \\
& \langle p, p\rangle Q_{C}(\lambda)=\sum_{j=1}^{N} p_{j}^{2} \prod_{k=1, k \neq j}^{N}\left(\lambda-\lambda_{k}\right), \quad K(\lambda)=\prod_{j=1}^{N}\left(\lambda-\lambda_{j}\right) .
\end{aligned}
$$


Соответствуюшим образом выбирая $N-1$ различных вешественных нулей $\mu_{1}^{B}, \ldots, \mu_{N-1}^{B}$ и $\mu_{1}^{C}, \ldots, \mu_{N-1}^{C}$ величин $Q_{B}(\lambda)$ и $Q_{C}(\lambda)$, получаем

$$
\begin{aligned}
Q_{B}(\lambda)=\prod_{j=1}^{N-1}\left(\lambda-\mu_{j}^{B}\right), \quad Q_{C}(\lambda)=\prod_{j=1}^{N-1}\left(\lambda-\mu_{j}^{C}\right), \\
\frac{\langle\Lambda q, q\rangle}{\langle q, q\rangle}=A_{1}-\sum_{k=1}^{N-1} \mu_{k}^{B}, \\
\frac{\langle\Lambda p, p\rangle}{\langle p, p\rangle}=A_{1}-\sum_{k=1}^{N-1} \mu_{k}^{C}, \\
\frac{\left\langle\Lambda^{2} q, q\right\rangle}{\langle q, q\rangle}=A_{1} \frac{\langle\Lambda q, q\rangle}{\langle q, q\rangle}-A_{2}+\sum_{k, j=1, j<k}^{N-1} \mu_{j}^{B} \mu_{k}^{B}, \\
\frac{\left\langle\Lambda^{2} p, p\right\rangle}{\langle p, p\rangle}=A_{1} \frac{\langle\Lambda p, p\rangle}{\langle p, p\rangle}-A_{2}+\sum_{k, j=1, j<k}^{N-1} \mu_{j}^{C} \mu_{k}^{C},
\end{aligned}
$$

где

$$
A_{1}=\sum_{j=1}^{N} \lambda_{j}, \quad A_{2}=\sum_{k, j=1, j<k}^{N} \lambda_{j} \lambda_{k} .
$$

Очевидно, уравнения (13)-(16) эквивалентны уравнениям

$$
\begin{gathered}
\sum_{k=1}^{N-1} \mu_{k}^{B}=A_{1}-\frac{\langle\Lambda q, q\rangle}{\langle q, q\rangle}, \\
\sum_{k=1}^{N-1} \mu_{k}^{C}=A_{1}-\frac{\langle\Lambda p, p\rangle}{\langle p, p\rangle}, \\
\left(A_{1}-\sum_{k=1}^{N-1} \mu_{k}^{B}\right)^{2}-\sum_{k=1}^{N-1}\left(\mu_{k}^{B}\right)^{2}=2 A_{2}-A_{1}^{2}+2 \frac{\left\langle\Lambda^{2} q, q\right\rangle}{\langle q, q\rangle}, \\
\left(A_{1}-\sum_{k=1}^{N-1} \mu_{k}^{C}\right)^{2}-\sum_{k=1}^{N-1}\left(\mu_{k}^{C}\right)^{2}=2 A_{2}-A_{1}^{2}+2 \frac{\left\langle\Lambda^{2} p, p\right\rangle}{\langle p, p\rangle} .
\end{gathered}
$$

С одной стороны, $u_{x}=-2\left\langle q, q_{x}\right\rangle=-2\langle q, \partial H / \partial p\rangle=-2\langle\Lambda q, q\rangle-2 u c_{0}(t)$, где функция $c_{0}(t)$ зависит только от $t$. Из уравнения (13), таким образом, получаем

$$
\frac{\partial}{\partial x} \ln u=2 A_{1}-2 \sum_{k=1}^{N-1} \mu_{k}^{B}-2 c_{0}(t) .
$$

С другой стороны, $u_{t_{2}}=-2\left\langle q, q_{t_{2}}\right\rangle=-2\left\langle q, \partial F_{2} / \partial p\right\rangle=-2\left\langle\Lambda^{2} q, q\right\rangle$. С учетом (17) это дает равенство

$$
\frac{\partial}{\partial t_{2}} \ln u=\left(A_{1}-\sum_{k=1}^{N-1} \mu_{k}^{B}\right)^{2}-\sum_{k=1}^{N-1}\left(\mu_{k}^{B}\right)^{2}-2 A_{2}+A_{1}^{2} .
$$


Отсюда имеем

$$
\begin{aligned}
u(x, t)= & u\left(x_{0}, t_{0}\right) \exp \left(\int_{t_{0}}^{t}\left[\left(A_{1}-\sum_{k=1}^{N-1} \mu_{k}^{B}\right)^{2}-\sum_{k=1}^{N-1}\left(\mu_{k}^{B}\right)^{2}-2 A_{2}+A_{1}^{2}\right] d t+\right. \\
& \left.+\int_{x_{0}}^{x}\left[2 A_{1}-2 \sum_{k=1}^{N-1} \mu_{k}^{B}-2 c_{0}(t)\right] d x\right), \quad t=t_{2},
\end{aligned}
$$

где $x_{0}$ и $t_{0}$ - два фиксированных начальных значения. Аналогичным образом $v(x, t)$ имеет представление

$$
\begin{aligned}
v(x, t)= & v\left(x_{0}, t_{0}\right) \exp \left(-\int_{t_{0}}^{t}\left[\left(A_{1}-\sum_{k=1}^{N-1} \mu_{k}^{C}\right)^{2}-\sum_{k=1}^{N-1}\left(\mu_{k}^{C}\right)^{2}-2 A_{2}+A_{1}^{2}\right] d t-\right. \\
& \left.-\int_{x_{0}}^{x}\left[2 A_{1}-2 \sum_{k=1}^{N-1} \mu_{k}^{C}-2 c_{0}(t)\right] d x\right), \quad t=t_{2} .
\end{aligned}
$$

Поскольку уравнения (18) и (19) задают решение нелинейного солитонного уравнения (11), то для получения их явного вида нам нужно вычислить только четыре ключевых выражения $\sum_{k=1}^{N-1}\left(\mu_{k}^{J}\right)^{k}, J=B, C, k=1,2$. Будем следовать подходу для случая решетчатого уравнения Тоды [10], [11]. Для двух наборов координат Дарбу $\mu_{j}^{J}, J=B, C$, $j=1, \ldots, N-1$, имеем ключевые равенства

$$
\sum_{j=1}^{N-1}\left(\mu_{j}^{J}\right)^{k}=C_{k}(\Gamma)-\sum_{s=1}^{2} \operatorname{Res}_{\lambda=\infty_{s}} \lambda^{k} d \ln \Theta\left(A(P)-\phi-K_{J}\right), \quad J=B, C, \quad k=1, \ldots, N-1,
$$

где $C_{k}(\Gamma)$ - константа [10], [12], определяемая только компактной римановой поверхностью $Г$ рода $N-1, \mu^{2}=P(\lambda) K(\lambda)$,

$$
\begin{aligned}
P(\lambda) & =K(\lambda)+\sum_{j=1}^{N} E_{j} \prod_{k=1, k \neq j}^{N}\left(\lambda-\lambda_{k}\right), \\
\infty_{1} & =\left(0,\left.\sqrt{P\left(z^{-1}\right) K\left(z^{-1}\right)}\right|_{z=0}\right) \\
\infty_{2} & =\left(0,-\left.\sqrt{P\left(z^{-1}\right) K\left(z^{-1}\right)}\right|_{z=0}\right)
\end{aligned}
$$

$A(P)=\int_{P_{0}}^{P} \omega$ является отображением Абеля, в котором $P_{0}-$ произвольная фиксированная точка на $\Gamma, \omega=\left(\omega_{1}, \ldots, \omega_{N-1}\right)^{\mathrm{T}}$,

$$
\omega_{j}=\sum_{l=1}^{N-1} r_{j, l} \widetilde{\omega}_{l}=\sum_{l=1}^{N-1} r_{j, l} \frac{\prod_{k=1, k \neq l}^{N}\left(\lambda-\lambda_{k}\right)}{2 \sqrt{K(\lambda) P(\lambda)}} d \lambda
$$

есть нормированная голоморфная дифференциальная форма, а $r_{j, l}$ - нормировочный множитель. Далее, $j$-я компонента $\phi_{j}(x, t)(N-1)$-мерного вектора $\phi$ равна

$$
\sum_{l=1}^{N-1} r_{j, l}\left(Q_{l}^{0}+\frac{\lambda_{l} x}{2}+\frac{\lambda_{l}^{2} t}{2}+C_{l}(t)+\widetilde{C}_{l}(x)\right)
$$


при произвольной постоянной $Q_{l}^{0}$ и произвольных функциях $C_{l}(t), \widetilde{C}_{l}(x) \in C^{\infty}(R)$. Векторы $K_{B}$ и $K_{C}$ из $\mathbb{C}^{N-1}$ являются двумя векторами римановых констант, связанных с соответствуюшими координатами Дарбу $\mu_{j}^{B}$ и $\mu_{j}^{C}$. Риманова тета-функция $\Theta(\xi)$ [13] определена на римановой поверхности $\Gamma$.

Вычисляя вычет в точках $\infty_{s}, s=1,2$, при $k=1,2$ находим

$$
\begin{aligned}
\sum_{j=1}^{N-1} \mu_{j}^{J} & =C_{1}(\Gamma)-\frac{\partial}{\partial x} \ln \frac{\Theta_{1}^{J}}{\Theta_{2}^{J}}, \\
\sum_{j=1}^{N-1}\left(\mu_{j}^{J}\right)^{2} & =C_{2}(\Gamma)+\frac{\partial}{\partial t} \ln \frac{\Theta_{1}^{J}}{\Theta_{2}^{J}}-\frac{\partial^{2}}{\partial x^{2}} \ln \Theta_{1}^{J} \Theta_{2}^{J},
\end{aligned}
$$

где

$$
\Theta_{s}^{J}=\Theta\left(\phi+K_{J}+\eta_{s}\right), \quad J=B, C,
$$

$$
\eta_{s, j}=\int_{\infty_{s}}^{P_{0}} \omega_{j}, \quad s=1,2,
$$

есть $j$-я компонента $(N-1)$-мерного вектора $\eta_{s}$.

Подставляя приведенные выше равенства в (18) и (19) и сортируя их, получаем явные решения солитонного уравнения (11):

$$
\begin{aligned}
u(x, t)= & \left.\left.u\left(x_{0}, t_{0}\right) e^{a\left(t-t_{0}\right)+2\left(b-c_{0}\right)\left(x-x_{0}\right)} \frac{\Theta_{1}^{B}}{\Theta_{2}^{B}}\right|_{t=t_{0}}\left(\frac{\Theta_{2}^{B}}{\Theta_{1}^{B}}\right)^{2}\right|_{x=x_{0}} \times \\
& \times \frac{\Theta_{1}^{B}}{\Theta_{2}^{B}} \exp \left(\int_{t_{0}}^{t}\left[\frac{\partial^{2}}{\partial x^{2}} \ln \Theta_{1}^{B} \Theta_{2}^{B}+\left(b+\frac{\partial}{\partial x} \ln \frac{\Theta_{1}^{B}}{\Theta_{2}^{B}}\right)^{2}\right] d t\right), \\
v(x, t)= & \left.\left.v\left(x_{0}, t_{0}\right) e^{-a\left(t-t_{0}\right)-2\left(b-c_{0}\right)\left(x-x_{0}\right)} \frac{\Theta_{2}^{C}}{\Theta_{1}^{C}}\right|_{t=t_{0}}\left(\frac{\Theta_{1}^{C}}{\Theta_{2}^{C}}\right)^{2}\right|_{x=x_{0}} \times \\
& \times \frac{\Theta_{2}^{C}}{\Theta_{1}^{C}} \exp \left(\int_{t_{0}}^{t}\left[\frac{\partial^{2}}{\partial x^{2}} \ln \Theta_{2}^{C} \Theta_{1}^{C}+\left(b+\frac{\partial}{\partial x} \ln \frac{\Theta_{2}^{C}}{\Theta_{1}^{C}}\right)^{2}\right] d t\right),
\end{aligned}
$$

где $a=A_{1}^{2}-C_{2}(\Gamma)-2 A_{2}$ и $b=A_{1}-C_{1}(\Gamma)$ - две постоянные, $c_{0}=c_{0}(t) \in C^{\infty}(R)$ является заданной функцией $t$, а $x_{0}$ и $t_{0}$ - начальные значения. В результате приходим к следуюшей теореме.

Теорема 4. Уравнения АКНС (11) имеют пару явных решений (20) и (21), заданных видом римановой тета-функиии и называемых алгебро-геометрическим решением.

Аналогичный процесс вычисления может привести также к алгебро-геометрическому решению АКНС-уравнений высших порядков (9), но мы не рассматриваем здесь этот более сложный случай. 


\section{6. ЗАКЛЮЧЕНИЕ}

Мотивация данных исследований исходит из наших работ по конечномерным интегрируемым системам, описанным в [10]. Действительно, на примере уравнений АКНС мы показали, что процедура алгебро-геометрического решения успешно распространяется с конечномерных интегрируемых систем на интегрируемые НЭУ, или солитонные уравнения. Эту процедуру можно применить и к другим НЭУ. Жу [12] привел алгебро-геометрическое решение уравнения Жоляна-Миодека. Вслед за тем Жанг [14] и Ду [15] также получили алгебро-геометрические решения некоторых солитонных уравнений, используя этот метод. Разумеется, сушествуют и другие методы решения солитонных уравнений. Недавно Дайфт, Итс и Жу [16] с использованием асимптотического метода Римана-Гильберта получили $\Theta$-функциональные решения некоторых НЭУ, таких как Кд $\Phi$, мКдФ и нелинейное уравнение Шредингера. Все эти методы до сих пор находятся в состоянии развития.

Благодарности. Автор искренне благодарит Fachbereich 17 в Университете-GH Kassel и в особенности профессора Страмппа и профессора Фарнхорна за их любезное приглашение и гостеприимство.

Работа была поддержана со стороны Research Program Лос-Аламосской Национальной Лаборатории (США), немецкого фонда Александра Гумбольдта, китайского национального фундаментального исследовательского проекта "Нелинейная наука", Специального гранта за выдаюшуюся китайскую Ph.D. диссертацию и Doctoral Program Foundation Министерства образования Китая.

\section{Список литературы}

[1] M. J. Ablowitz, D. J. Kaup, A. C. Newell, H. Segur. Phys. Rev. Lett. 1973. V. 31. P. 125.

[2] M. J. Ablowitz, D. J. Kaup, A. C. Newell, H. Segur. Stud. Appl. Math. 1974. V. 53. P. 249.

[3] M. Абловии, X. Сигур. Солитоны и метод обратной задачи рассеяния. М.: Мир, 1987.

[4] P. D. Lax. Commun. Pure Appl. Math. 1975. V. 28. P. 141.

[5] Б. А. Дубровин, И. М. Кричевер, С. П. Новиков. ДАН СССР. 1976. Т. 229. С. 15.

[6] В. Е. Захаров, А. Б. Шабат. ЖЭТФ. 1972. Т. 61. № 1. С. 118.

[7] C. W. Cao. Sci. China A (Chinese Edition). 1989. V. 32. P. 701; Sci. Sin. A (English Edition). 1990. V. 33. P. 528

[8] Л.А. Тахтаджсян, Л. Д. Фаддеев. Гамильтонов подход в теории солитонов. М.: Наука, 1986.

[9] C. W. Cao. Acta Math. Sin. New Series. 1991. V. 7. P. 216.

[10] Z. J. Qiao. Generalized Lax algebra, $r$-matrix, and algebraic-geometric solution for the integrable system. Doctoral dissertation. Fudan University, China, 1997.

[11] Z. J. Qiao. Chin. Sci. Bull. 1999. V. 44. P. 114.

[12] R. G. Zhou. The finite-dimensional integrable systems related to the soliton equations. Doctoral dissertation. Fudan University, China, 1997.

[13] Д. Мамфорд. Лекции о тэта-функциях. М.: Мир, 1984.

[14] J. S. Zhang. The generating functional method for finite-dimensional integrable systems. Doctoral dissertation. Zhengzhou University, China, 1998.

[15] D. L. Du. Integrability of the nonlinearized eigenvalue problems under the Lie-Poisson structure. Doctoral dissertation. Zhengzhou University, China, 1998.

[16] P. A. Deift, A.R. Its, X. Zhou. Ann. Math. 1997. V. 146. P. 149. 